\title{
A NEW DIRECTIONAL ACOUSTIC LENS: V-GROOVE LENS
}

\author{
A. Bozkurt, G. Yaralığlu, A. Atalar and H. Köymen \\ Bilkent University, Ankara, Turkey 06533
}

\begin{abstract}
A new directional acoustic lens is introduced. The geometry is very similar to the line-focus lens except the lens cavity, which is shaped as a groove with flat-bottom $V$ cross section. The slanted planar edges of the groove are inclined in order to generate waves incident on the object surface at a critical angle. Hence, the edges of the groove act like two wedge transducers facing each other. The cross section of the lens is the same as that of the Lamb Wave Lens. Therefore, it enjoys the same sensitivity to surface wave excitations. On the other hand, since the cross section remains the same along one of the lateral directions, it has directional properties very similar to that of the Line Focus Beam Lens. The waves normally incident on the object surface generated from the flat-bottom, interfere with those at the critical angle, giving rise to a $\mathrm{V}(\mathrm{Z})$ effect. Calculated responses of the lens are presented for silicon (001) surface as a function of crystal orientation. The calculated curves are compared with measurement results. The leaky wave velocities are extracted from the measurement results using the conventional FFT algorithm. A new model based algorithm is proposed for extracting the velocity information from $V(Z)$ data.
\end{abstract}

\section{Introduction}

A directional lens such as line focus beam (LFB) lens [1] produce a $V(Z)$ pattern from which surface wave velocity in a particular direction can be deduced with high accuracy. The accuracy depends on the number of oscillations in the pattern. For high velocity materials the period is large, and thus there will be a limited number of oscillations, resulting in a low measurement accuracy. Moreover, the signal level in $V(Z)$ gets smaller as the defocus distance is increased.

The conventional spherical lens was originally designed for imaging purposes. After it is discovered that $V(Z)$ has unique material characterization properties, this lens is used mostly for characterization despite its deficiency in exciting leaky waves. Lamb wave lens combines the $V(Z)$ effect with its higher efficiency of leaky wave excitation. It maintains a large signal level at an extended defocus range. However, it does not have a direction sensitivity which is required for the characterization of anisotropic materials.

In this paper, we introduce a new directional lens, $V$-groove lens, with a higher sensitivity as compared to other directional lenses. It inherits the high efficiency of the Lamb wave lens, while providing the high directionality of the LFB lens. First, the geometry of the V-groove lens is described and design considerations are discussed. Then, the response of the lens as a function of defocus distance is investigated. Theoretical simulations are performed for an anisotropic crystal to deduce the accuracy of such a lens. Experimental results are presented and compared with the theory. Moreover, a refinement in the procedure of extracting velocity information from $V(Z)$ data is presented. The new procedure involves a model based analysis approach rather than a simple FFT analysis on the data.

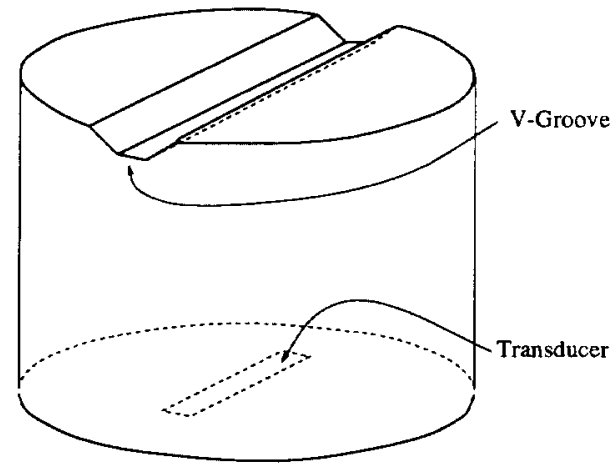

Figure 1: Geometry of the V-groove lens

\section{V-Groove Lens}

$V$-groove lens differs from the line-focus-beam lens in the way the refracting element of the lens is fabricated. As opposed to the cylindrical cavity of the LFB lens, V-groove lens has a $\mathrm{V}$ shaped groove with flattened bottom as shown in Fig. 1. Essentially, the relationship between the LFB lens and the conventional lens is the same as the relationship between $\mathrm{V}$ groove lens and the Lamb wave lens [2]. In other words, leaky waves are selectively excited on the surface of the object when V-groove lens is used, while, in case of LFB lens, all of the modes are excited due to its wide angular spectrum.

The transducer is designed so as to insonify a substantial portion of the groove with minimal waste of power elsewhere. The flat bottom part does not cause any refraction, and thus a part of the incident beam insonifies the object surface at normal incidence. Symmetrical sides of the groove causes a 
refraction, and hence two symmetrical beams insonify the object surface at the same incidence angle. The interference of the refracted beams, which encounter leaky wave modes on the object surface, with the reference beam resulting from the specular reflection of the normally incident beam produces the $V(Z)$. A good match between median direction of the refracted beam and the critical angle for the object improves the measurement accuracy.

\section{Simulations and Experiments}

Simulating the performance of a lens involves propagation of acoustic waves between the transducer and the refracting element. The wavefront is then propagated through the refracting element using ray theory. The wave front is then reflected from object surface upon propagation in liquid. This analysis is similar to the one developed for Lamb wave lens [2], except for the circular symmetry. While the circular symmetry of the Lamb lens allows the use of fast Hankel transform for propagation purposes, propagation problem in $\mathrm{V}$-groove lens requires the more costly two dimensional FFT. Also, the calculation of the reflection coefficient at a liquid anisotropic solid interface is considerably more complicated [3].

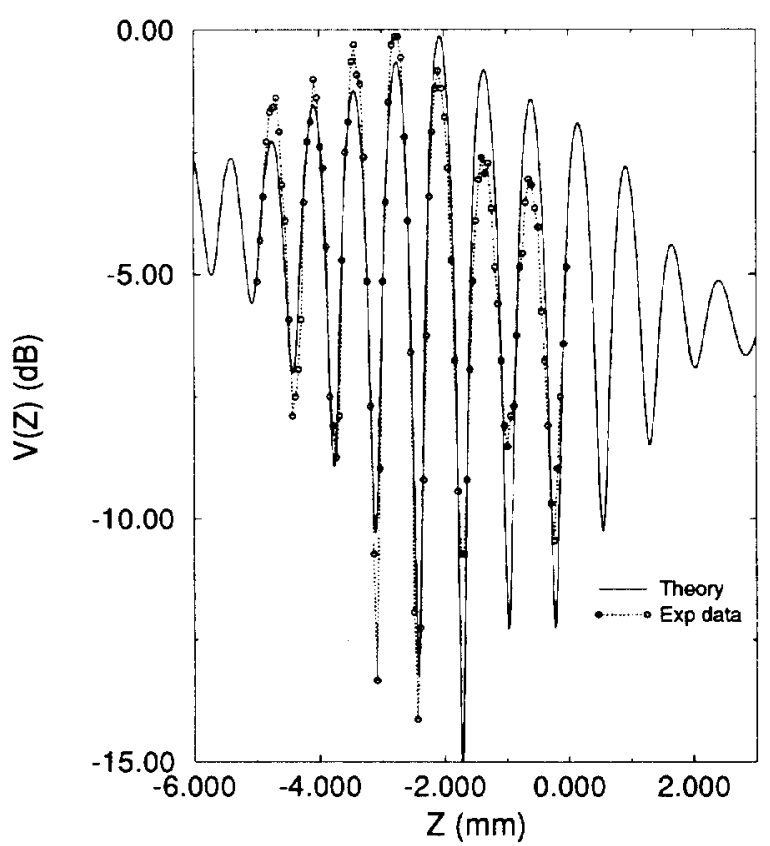

Figure 2: Calculated and measured $V(Z)$ values along [100] direction on (001) surface of Si at $f=25 \mathrm{MHz}$

A $V$-groove lens is designed to measure surface acoustic wave velocity on silicon. Lens operates at $25 \mathrm{MHz}$. The silicon sample is a 525 microns thick silicon wafer. The Rayleigh wavelength is approximately 200 microns, and hence the wafer thickness is large enough for measurement purposes. Water temperature was stable within 0.2 degrees. The alignment of the lens is achieved easily by maximizing the signal from the flat part of the lens, since the maximum signal is reached when the object surface is perfectly parallel to the flat part of V-groove. A simulation is done for this lens for the (001) surface of silicon as the reflector. The resulting $V(Z)$ curve is depicted in Fig. 2 together with the measurements along [100] direction. The $V(Z)$ simulations are repeated along varying directions for the same surface of silicon.

\section{Leaky wave velocity extraction}

The conventional procedure [4] adapted for extracting SAW velocity from measured $V(Z)$ data can be summarized as follows:

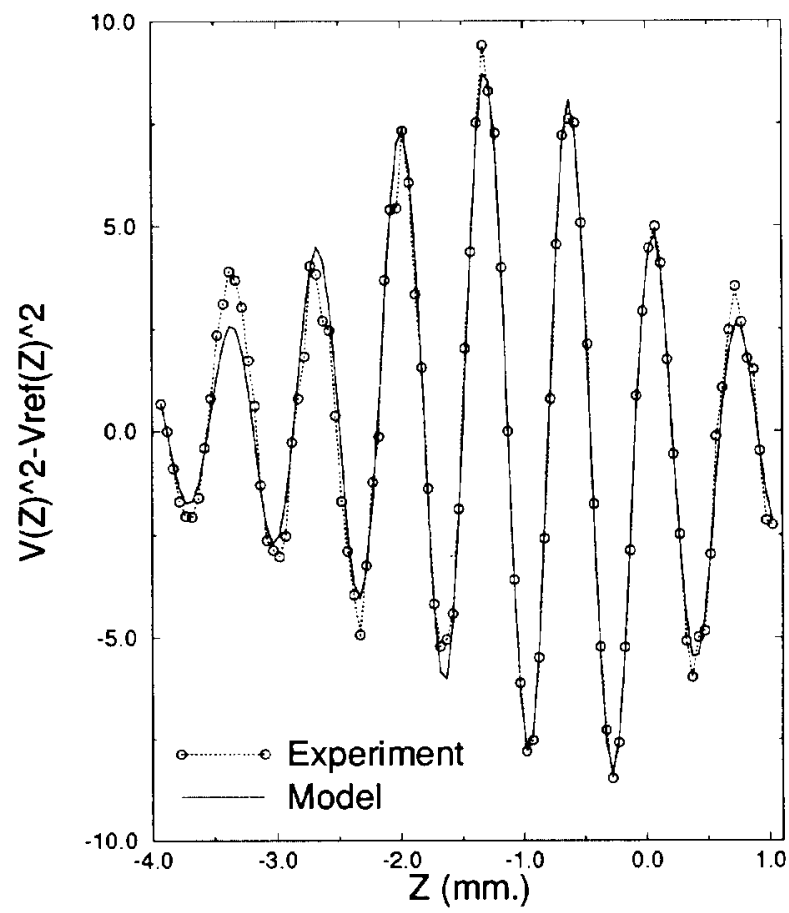

Figure 3: Experimental data points $V^{2}(Z)-V_{\text {ref }}^{2}(Z)$ for $(001)$ surface of $\mathrm{Si}(F=25 \mathrm{MHz})$ and least squared fitted curve

- Measure $V(Z)$ for the object

- Obtain a $V_{r e f}(Z)$ using an object with no leaky wave generation at the $V$-groove excitation range.

- Find $V^{2}(Z)-V_{\text {ref }}^{2}(Z)$

- Filter out any unwanted interference frequencies

- Pad data with zeros 
- Use a proper window function

- Apply FFT to find the period of oscillation

- Determine velocity from period

This method yields its best results if there is only one leaky wave mode. It is difficult to get accurate results particularly when there are two modes with close velocities. Unfortunately, many anisotropic materials support pseudo surface waves along particular directions [5] with a velocity very close to the SAW velocity. FFT algorithm gives biased results in such a case. To alleviate this problem, an alternative procedure is proposed. A model based algorithm [6] is adopted, which suits better to the physical nature of the $V(Z)$ :

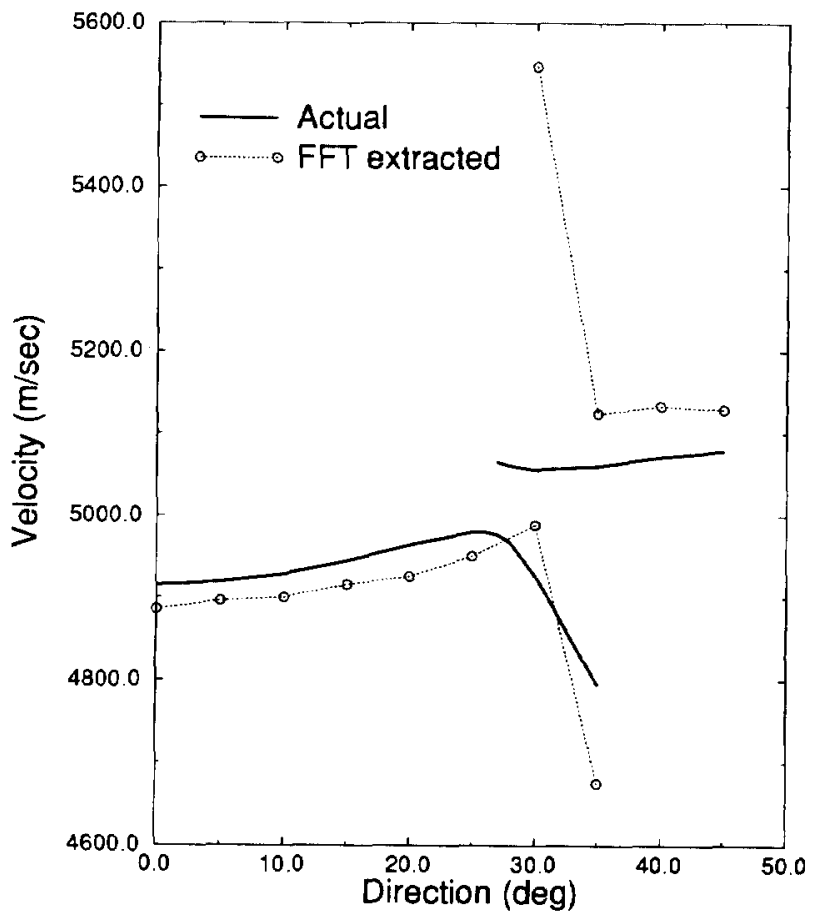

Figure 4: Calculated and measured velocity (using FFT method) values on (001) surface of $\mathrm{Si}$ as a function of direction

- Measure $V(Z)$ for the object

- Obtain a $V_{r e f}(Z)$ as above

- Fit the model parameters to $V_{\text {ref }}(Z)$ in the least mean square sense, using Nelder-Meade simplex search.

- Find $V^{2}(Z)-V_{r e f}^{2}(Z)$

- Fit the model parameters using the same algorithm to the squared difference. Find the period of oscillation.

- Determine velocity from period
A simple model is selected to reduce the computational complexity of the search process. The model assumes uniform insonification of the $\mathrm{V}$-groove and ignores the diffraction in the liquid. The output voltage is obtained as the absolute value of a sum of three complex terms. The first term is due to the flat central portion of the $V$-groove lens and its phase is assumed to change linearly with the defocus distance. The second term arises from the specular reflection of obliquely incident waves. The change in its phase depends on the inclination angle of the $\mathrm{V}$-groove as well as the defocus distance. Variation in its amplitude is determined from geometrical considerations. The last term exists only if a leaky wave is excited on the object surface. The phase of this term is assumed to depend on the critical angle of the object material. Since leaky waves are assumed to decay exponentially [7], the amplitude of the third term is exponentially dependent on the defocus distance. Copper is used as the reference material, since no leaky wave can be excited on its surface by this lens. Using the reference material data, $V_{\text {ref }}(Z)$, the unknown parameters in the first and second term are determined. The parameters of the third term -among them is the leaky wave velocity of the object- is subsequently determined from the measured $V(Z)$ data on the object. Fig. 3 shows the measured squared difference data together with the results of the simple model whose parameters are optimized to best fit the data.

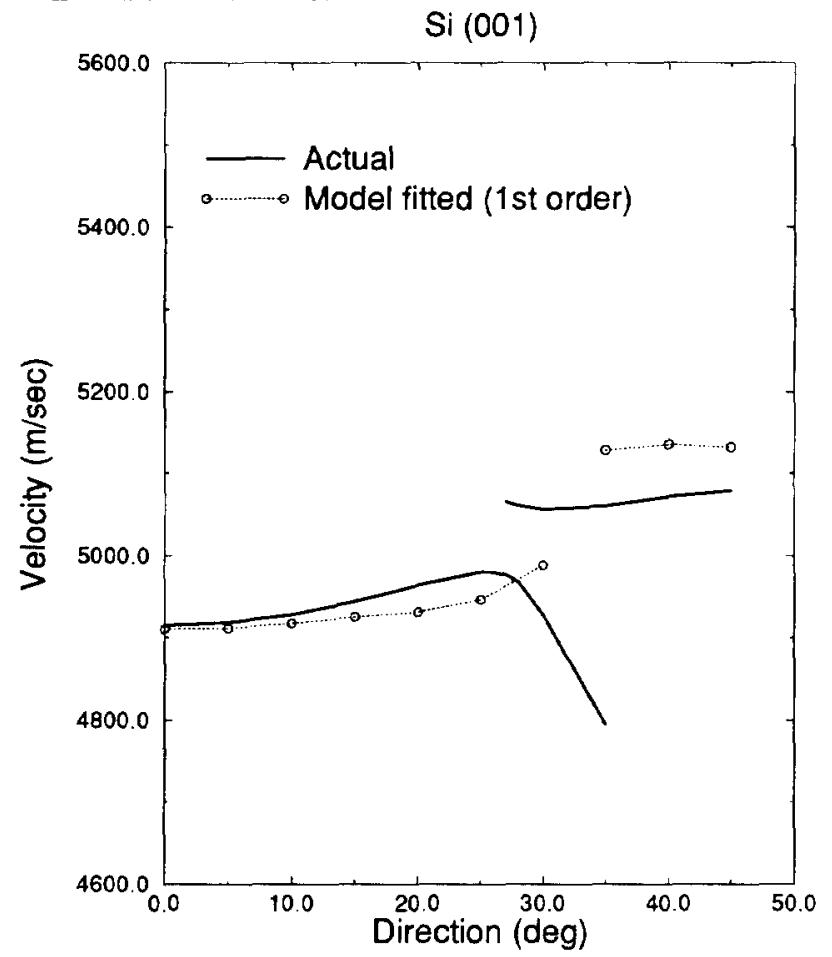

Figure 5: Calculated and measured velocity (using model fitting method) values on (001) surface of $\mathrm{Si}$ as a function of direction 
The velocity is extracted from the measured $V(Z)$ data for (001) surface of silicon along different directions using FFT method and the proposed method. These experimental results are given in Fig. 4 along with calculated leaky wave velocities from elastic constants [8]. The estimated velocity values follows the variation predicted from tabulated elastic constants within $1 \%$. It is interesting to note that for angles larger 25 degrees, presence of pseudo surface waves is indicated by an emerging extra peak in the frequency spectrum. However, the accuracy at points where the two modes are of equal magnitude seems to be degraded.

The velocity estimates obtained from the same experimental data using model fitting method is depicted in Fig. 5. The model assumes a single leaky wave excitation. It can be observed that the agreement between the extracted values and the calculations are significantly better, when there is no pseudo surface wave excitation. Obviously, in the presence of pseudo surface waves, a single model order can not match the inherent complexity of the data. Hence, the estimated velocity value falls somewhere between the two modes.

\section{Conclusions}

The proposed V-groove lens is proved to be a powerful tool for measuring leaky velocities with a good directional sensitivity. Directional properties is comparable to LFB lens while its leaky wave excitation efficiency is as good as the Lamb wave lens. Since its excitation angle is fixed, a given $V$-groove lens can only be used for a limited range of velocities. Typically, for a particular material a matching V-groove lens must be used. Because of its high excitation efficiency, the modulation index in the $V(Z)$ curve maintains a high value over an extended defocus distance. This provides a high signal-to-noise ratio. Hence, a more accurate characterization is possible.

The best use of $V(Z)$ data requires a processing procedure which concur with nature of the physical problem. The traditional approach is to use a FFT based spectral algorithm to find the velocities. This method yields particularly erroneous results if there are more than one leaky wave excitation. The method proposed in this paper adopts a model based approach to extract velocity. The model makes use of all available physical and geometrical data with some unknown parameters. The unknown parameters are found at the end of a minimization procedure which fits the measured data to the model prediction. The performance of the $\mathrm{V}$ groove lens used along with the new extraction procedure is experimentally tested on silicon. Characterization of silicon has inherent difficulties because of its relatively high SAW velocity.

As long as the surface wave velocity remains within the range dictated by the fixed inclination angle of the V-groove lens, it is possible to estimate the surface wave velocity as a function of orientation with a very high accuracy.

\section{Acknowledgment}

This work is supported by Turkish Scientific and Technical Research Council, TUBITAK.

\section{References}

[1] J. Kushibiki and N. Chubachi "Material characterization by line-focus-beam acoustic microscope," IEEE Trans. Sonics Ultrason, vol. 32, pp. 189-212, 1985.

[2] A. Atalar and H. Köymen "A high efficiency lamb wave lens for subsurface imaging," in Proc. of IEEE $1989 \mathrm{Ul}$ trasonics Symposium, pp. 813-816, 1989.

[3] O. Arıkan, E. Teletar, and A. Atalar "Reflection coeficient null of acoustic waves at a liquid-anisotropic-solid interface," J. Acoust. Soc. Am., vol. 85, pp. 1-10, 1989.

[4] A. Briggs. Acoustic Microscopy. Oxford Press, Oxford, 1992.

[5] T.C. Lim and G.W. Farnell "Character of pseudo surface waves on anisotropic crystals," J. Acoust. Soc. Am., vol. 45 , pp. $845-851,1969$.

[6] S.L. Marple Jr, Digital Spectral Analysis with Applications. Prentice-Hall, Englewood Cliffs, 1987.

[7] H.L. Bertoni and T. Tamir "Unified theory of rayleigh angle phenomena for acoustic beams at liquid--solid interfaces," Appl. Phys., vol. 2, pp. 157-172, 1973.

[8] B.A. Auld. Acoustic Fields and Waves in Solids, volume 1. Wiley, New York, 1973. 